\title{
Corpus
}

CORPUS

$20 \mid 2020$

Corpus complexes

\section{Les corpus de la communication médiée par les réseaux : une introduction}

Céline Poudat, Ciara R. Wigham and Loïc Liégeois

\section{(2) OpenEdition \\ 1 Journals}

\section{Electronic version}

URL: http://journals.openedition.org/corpus/4720

DOI: $10.4000 /$ corpus. 4720

ISSN: 1765-3126

\section{Publisher}

Bases; corpus et langage - UMR 6039

\section{Electronic reference}

Céline Poudat, Ciara R. Wigham and Loïc Liégeois, « Les corpus de la communication médiée par les réseaux : une introduction », Corpus [Online], 20 | 2020, Online since 31 January 2020, connection on 10 December 2020. URL : http://journals.openedition.org/corpus/4720 ; DOI : https://doi.org/

$10.4000 /$ corpus. 4720

This text was automatically generated on 10 December 2020 .

(c) Tous droits réservés 


\title{
Les corpus de la communication médiée par les réseaux : une introduction
}

\author{
Céline Poudat, Ciara R. Wigham and Loïc Liégeois
}

1 Si le développement du web a rendu accessibles des masses de données numériques, facilitant la collecte de textes et le développement de corpus, il a également donné naissance à de nouveaux genres qui défient les représentations, les méthodes et les grilles d'analyses développées jusqu'à présent. Ainsi a-t-on vu apparaître des corpus assez éloignés des premiers corpus écrits traditionnels, regroupés sous la bannière de la CMR (Communication Médiée par les Réseaux / Computer-Mediated Communication ou CMC en anglais). Déterminée par des dispositifs sociaux qui intègrent des particularités communicatives inédites, la CMR est un objet singulièrement complexe à appréhender, qui a donné lieu à des analyses nombreuses, tant du point de vue des sciences sociales que de celui des études linguistiques. Un champ pluridisciplinaire focalisé sur ces objets particuliers s'est même développé (Herring, 1996, 2001 ; voir les revues Journal of Computer-Mediated Communication, Language@Internet).

2 Le présent numéro s'intéresse à la CMR écrite du point de vue particulier des corpus : comment regrouper les données de la CMR en corpus et selon quels principes de constitution? Comment segmenter les données? Quels standards mobiliser pour représenter les interactions de la CMR? Qu'est-ce que les corpus peuvent nous apprendre de la CMR?

3 Constituer un corpus de CMR nous semble être une opération particulièrement ardue, peut-être d'abord du fait de la particularité de la situation communicative, dont nous n'avons jamais eu autant de traces matérielles: chaque message est généralement signé, muni de son horodatage (timestamp) et peut être précisément situé dans l'espace de discussion (fil, page, topic le cas échéant); selon les spécificités du dispositif de communication et la nature publique ou privée des messages publiés, on peut disposer de l'historique complet ou partiel des interactions entre les contributeurs, ce qui permet techniquement d'éclairer chaque interaction avec des éléments longitudinaux 
et mémoriels parfois anciens et nombreux, ce qui est encore une fois tout à fait inédit ; enfin, lorsque les interactions ont une visée communicative explicite et sont adossées à un objet donné qu'elles commentent, qu'elles évaluent ou qu'elles développent, l'objet extralinguistique en question peut être extrait, observé et parfois explicitement articulé aux messages s'ils en commentent certains aspects (ex. documents collaboratifs, qu'ils soient par exemple du texte ou du code informatique). Ce foisonnement des traces de la situation d'énonciation des messages est tout à la fois stimulant du fait de son caractère inédit, et inhibant car le choix des métadonnées n'a jamais été aussi difficile. C'est certainement ce qui explique la prépondérance des études plus manuelles et plus qualitatives de la CMR, qui analysent les données dans leur environnement natif et ne constituent d'ailleurs pas systématiquement de corpus de travail.

Dans tous les cas, la construction du corpus dépend de la visée de la recherche et les différents corpus de CMR présentés dans ce volume illustrent cette évidence; ainsi, le corpus de Panckhurst et al. (ce volume), de même que l'ensemble des sous-corpus de SMS représentés dans le corpus CoMeRe (COmmunication MEdiée par les REseaux, CoMeRe Repository (2014), voir Wigham et Poudat, ce volume), ne sont pas structurés en interactions puisqu'il s'agit d'étudier les spécificités du langage SMS, et non d'analyser comment les interactions se déploient dans un dispositif SMS. Il en va de même des corpus de Longhi (ce volume), qui explore les tweets comme des microtextes politiques, s'intéressant à l'usage de certains concepts ciblés en lien avec les comptes politiques qui les ont produits. En revanche, si l'analyste s'inscrit dans un cadre interactionnel ou pragmatique, il lui sera indispensable de considérer l'interaction elle-même pour être en mesure d'interpréter un message particulier. Or, la structuration de ces messages en interactions est généralement complexe, notamment du fait de la multiplicité des locuteurs susceptibles d'interagir dans des temporalités d'ailleurs variables sur ces fils. Les forums sont par exemple bien connus pour le nombre de conversations parallèles qu'ils rassemblent au sein d'un même topic (Marcoccia, 2004, Mangenot, 2008). Dans la plupart des corpus de la CMR, savoir à qui s'adresse un message particulier ou à qui il répond est une réelle gageure, les locuteurs ne l'explicitant pas systématiquement malgré les affordances des dispositifs. Dans un tel cadre, le retour au contexte natif reste ainsi crucial pour permettre à l'analyste d'élaborer son interprétation.

Une autre difficulté que posent les corpus de la CMR a trait à l'hybridité des données, qui nécessitent des (pré-)traitements parfois lourds et complexes. Deux niveaux d'hybridité nous semblent remarquables : (i) l'hybridité du discours employé, souvent décrit à mi-chemin entre oral et écrit, générateur de néologismes et de formes inédites, à tel point qu'on parle régulièrement de langue ou de langage SMS, clavardage, etc. Cette particularité entraîne tant des problèmes de représentation que d'indexation des données et on peut être amené à réaliser un appariement du texte avec une couche en français standard pour faciliter les requêtes et les traitements (voir Panckhurst, ce numéro, ou encore Kogkitsidou 2018, qui a développé un système de traduction automatique des SMS en français standard dans le cadre du projet SMS Alpes); (ii) l'hybridité des genres de la CMR, qui sont encore souvent instables à tel point qu'on peine à les désigner autrement que par le dispositif de communication médiatisée sur lesquels ils se fixent ; dans certains cas, le genre sera désigné par le message lui-même (le tweet, le SMS, le courriel) tandis que dans d'autres, ce sera le fil d'interaction (le clavardage), voire le dispositif lui-même qui fera office de dénomination générique (le 
forum, le blog). Ces remarques ne sont pas sans lien avec les différences de dénomination des messages des corpus du projet CoMeRe (Wigham et Poudat, ce numéro). De manière générale, la dénomination des genres renvoie aux genres écrits existants sur lesquels se sont éventuellement positionnés ces nouveaux genres: la lettre pour le courriel, le journal intime pour certains blogs, la conversation ordinaire ou spécialisée pour le forum, etc.

Ces singularités de la CMR bouleversent nos pratiques d'exploration de corpus (Poudat et Landragin, 2017 ; Lebart, Pincemin et Poudat, 2019) sur au moins deux plans : (i) celui de la caractérisation de ces genres, et des corpus de contrastes à convoquer, qui doivent être rigoureusement pensés : si l'on confronte souvent les interactions de la CMR aux formes de l'oral, on mobilisera également des corpus écrits existants comparables pour mettre en évidence les spécificités de ces nouvelles formes de communication. On pourra également, à un autre niveau, les comparer entre eux ; ces dispositifs, qui fédèrent des communautés d'utilisateurs qui se recouvrent, évoluent en résonance. Ils peuvent parfois se différencier ou au contraire s'agréger : les SMS sont par exemple concurrencés par les dispositifs de messagerie instantanée comme WhatsApp ou Facebook messenger et leur évolution programmée en RCS (Rich Communication Service) les y apparentent de plus en plus; (ii) la question de l'unité textuelle à considérer, et plus largement, de la représentation et de la structuration du corpus. On a vu que les dénominations des messages étaient susceptibles de varier d'un dispositif à l'autre, et qu'elles se spécialisaient d'autant plus que le genre s'édifiait sur les caractéristiques du message, et y référait dans sa dénomination. Par exemple, on parle de genre pour le tweet ou le SMS. Par rapport aux autres types de messages de la CMR, l'une des caractéristiques formelles de ces deux types de messages est certainement leur brièveté, du fait d'une contrainte technique de taille - le tweet est limité à 280 caractères, après avoir connu une limite à 140 caractères tandis que le SMS en compte 160 .

7 Le tweet, le SMS ou l'e-mail sont devenus des genres bien établis, qu'une majorité de locuteurs pratiquant la CMR sont en mesure d'identifier et de différencier - du moins ont-ils développé une représentation générique, avec un horizon d'attente et un ensemble prototypique de traits préférentiellement présents ou absents. Il est notable d'observer que l'interaction qui encadre le message produit semble secondaire, alors qu'elle est au cœur du clavardage, du forum ou de la discussion Wikipédia.

8 D'un genre de la CMR à l'autre, on s'interrogera ainsi différemment sur l'unité textuelle et sur les modalités de leur structuration. La question est loin d'être triviale en matière d'exploration de corpus, car l'analyste est toujours vigilant quant à ce qu'il compare. Est-il toujours pertinent et intéressant de contraster des messages entre eux à des fins typologiques? Doit-on plutôt considérer les interactions? Quels critères linguistiques prend-on en compte?

9 On voit ainsi à quel point on est loin des problématiques habituelles que posent les corpus écrits, et à quel point les pratiques d'exploration classiques des corpus textuels doivent être adaptées, et revisitées.

10 À travers les articles qu'il réunit, ce numéro propose donc une réflexion sur le corpus de communication médiée par les réseaux. Différents exemples de construction de corpus issus de médias sociaux différents seront exposés, tandis qu'une proposition de standardisation des corpus sera développée. Un fil rouge relie l'ensemble des textes qui composent ce numéro, en la personne de Thierry Chanier, à qui nous souhaitons rendre 
l'hommage qui lui revient. C'est en effet en grande partie sous son impulsion que ces problématiques de traitement et de standardisation des corpus se sont développées ces dernières années en France : c'est à lui que nous devons l'impulsion du projet national CoMeRe, grâce à son action au sein du consortium de linguistique Corpus écrits, aujourd'hui CORLI (CORpus, Langues et Interactions, consortium de la Très Grande Infrastructure de Recherche Huma-Num).

11 Le premier texte présenté dans ce numéro, rédigé par Céline Poudat, retrace donc le parcours de Thierry Chanier, en mettant notamment en lumière les avancées importantes que ses travaux ont permis de réaliser dans le champ de la didactique des langues, de la linguistique de corpus en général et de la CMR en particulier. Du projet Camille, qui avait permis de concevoir l'un des tout premiers CD-ROM de didactique du Français Langue Étrangère, au projet CoMeRe, les travaux les plus importants de Thierry Chanier sont restitués, laissant observer l'objectif qui les sous-tend : formaliser et standardiser la diversité et la complexité afin de faciliter le partage et la transmission des données et des savoirs scientifiques. D'ailleurs, Thierry Chanier ne dissociait jamais le travail de création d'un corpus linguistique (de son recueil et sa structuration jusqu'à sa diffusion) du travail d'analyse de ces mêmes données. Au contraire, il a invariablement souhaité mettre en avant, et cela depuis toujours et bien avant d'autres personnes du domaine, que le travail de constitution d'un corpus linguistique était une tâche qui relevait du linguiste et qui, lorsqu'elle était menée avec toute la rigueur scientifique nécessaire, se devait d'être reconnue et considérée comme un travail universitaire de premier plan.

12 C'est d'ailleurs dans ce cadre que le projet CoMeRe (Communication Médiée par les Réseaux) a été développé. Ce projet, que nous restituent Ciara R. Wigham et Céline Poudat, a en effet été dirigé par Thierry Chanier dans le but de constituer un corpus représentatif de la CMR, en agrégeant des corpus déjà existants et en en développant d'autres pour améliorer la représentativité de l'ensemble. Initié dans le cadre des activités du consortium Corpus Écrits de la TGIR Huma-Num, ce projet avait donc pour objectif de construire un corpus cohérent tout en veillant à prendre en considération toute leur diversité, et ce à plusieurs niveaux: les formes de communications médiatisées (écriture SMS, commentaires de blogs...), leur synchronicité (réception du message en temps réel pour un clavardage, en différé pour un commentaire sur un site internet) ou encore leur visée communicative (communication privée pour les SMS, argumentation publique pour une discussion Wikipedia, par exemple). Les deux auteures s'attachent ensuite à décrire la façon dont la réflexion autour des problématiques liées à la modélisation et à la structuration des données a eu lieu au sein du projet, pour mener, à terme, à la proposition d'un schéma de représentation suivant la structure générale de la Text Encoding Initiative (TEI). Une fois ce schéma présenté, les auteures offrent au lecteur une vue d'ensemble des retombées du projet. Parmi elles, nous noterons notamment l'élaboration et la pérennisation d'un schéma de représentation TEI des corpus de CMR.

13 Dans l'article suivant, Michael Beißwenger et Harald Lüngen se focalisent sur ce schéma XML qui suit le formalisme de base de la Text Encoding Initiative. Si, au départ, ce formalisme a été développé pour la représentation, la structuration et la pérennisation numérique de données issues de l'écrit (textes anciens, dictionnaires, manuscrits...) celui-ci a su s'adapter pour permettre la standardisation de données linguistiques diverses (corpus oraux et multimodaux, par exemple). Cette proposition 
de schéma de structuration des données a été développée dans le cadre d'une collaboration internationale par un groupe d'intérêt CMR de la TEI dont faisaient partie certains membres du projet CoMeRe et dont Thierry Chanier, à l'honneur dans ce numéro, était l'un des co-fondateurs. Le schéma proposé et détaillé par les auteurs vise à répondre à trois besoins essentiels de la communauté de chercheurs travaillant sur des données de CMR, à savoir : l'interopérabilité de corpus CMR hétérogènes structurés à partir de différents types de données, le besoin de structurer des interactions de différents genres en s'appuyant sur un schéma commun et la nécessité de représenter, de façon formelle et homogène, des données de langues variées dans le but de pouvoir élaborer des corpus comparables. Les auteurs proposent ainsi une réflexion sur les différents aspects à prendre en compte pour élaborer un tel modèle à partir de corpus hétérogènes et complexes, en particulier en raison de leur diversité et de la granularité des informations à structurer (informations sur l'émetteur, le contexte et le medium du message notamment). Ensuite, les auteurs se focalisent sur la description des propriétés des unités "basiques", c'est-à-dire des unités qu'ils estiment communes à toute interaction CMR. Cette description est illustrée au moyen d'exemples tirés de données provenant d'environnements CMR variés. En particulier, les auteurs s'attachent à décrire les particularités temporelles de la CMR et à expliquer comment certaines balises et attributs, déjà employés pour la structuration de corpus construits à partir de données de l'écrit, ont été spécifiquement adaptés à l'encodage de corpus de CMR (balises < post> et < div> et attribut @class notamment). Nous voyons ainsi comment la complexité des données de $\mathrm{CMC}$ a été prise en compte et gérée dans le but de proposer un module CMR de la TEI qui réponde aux demandes d'une communauté multidisciplinaire réunie autour de problématiques liées à l'analyse de données issues des médias sociaux.

Les trois articles qui suivent le travail de Michael Beißwenger et Harald Lüngen se focalisent d'ailleurs chacun sur l'analyse de données issues de médias sociaux différents. Tout d'abord Rachel Panckhurtst, Cédric Lopez et Mathieu Roche se focalisent dans leur contribution sur les SMS en revenant sur le projet Sud4science. Ce travail, développé dans le cadre du projet international sms4science, avait notamment pour objectif de recueillir, sur une période de treize semaines, un jeu de SMS échangés par des habitants de l'agglomération de Montpellier. Le but principal était en effet de collecter un jeu de données qui permettent, une fois structuré en corpus, de contribuer à l'étude de la communication par SMS et du langage qu'elle véhicule. Dans leur papier, les auteurs résument tout d'abord les différentes étapes dans la constitution du corpus 88milSMS. Dans la première partie de leur travail, les auteurs détaillent les étapes de structuration des données, de la méthodologie mise en place pour leur recueil des données jusqu'aux étapes de nettoyage et d'anonymisation du corpus. Les problématiques linguistiques liées à la structuration de ces données complexes et particulières sont ensuite abordées. Les auteurs insistent notamment sur les enjeux et les choix épistémologiques liés au transcodage des données issues des SMS en français standard et à l'annotation de phénomènes dits "non standards » dans un corpus de grande taille. Dans la deuxième partie de leur article, les auteurs présentent ensuite une synthèse des résultats scientifiques des chercheurs de l'équipe en adoptant une double perspective : celle du domaine des sciences du langage et celle du Traitement Automatique des Langues (TAL). La dernière partie de l'article est originale et vise à analyser la réutilisation du corpus. En effet, l'un des objectifs de la formalisation, de la pérennisation et du partage des corpus est bien évidemment de permettre leur 
réutilisation. Leur corpus ayant été déposé sur la plateforme de l'Équipement d'Excellence Ortolang (Panckhurst et al, 2016; ORTOLANG, 2019), celui-ci a pu être utilisé par la communauté. Les auteurs reviennent sur les résultats de deux sondages visant à mieux comprendre comment le corpus était réutilisé, et dans quel objectif scientifique. Leur étude met notamment en évidence l'un des aspects positifs du partage des corpus, à savoir l'appropriation et l'analyse des données par des chercheurs issus de domaines divers et parfois très éloignées de celui des sciences du langage comme la géographie ou la psychologie par exemple.

L'article suivant, rédigé par Julien Longhi, se focalise quant à lui sur un autre média social, à savoir Twitter. Plus précisément, la contribution de l'auteur vise à décrire et analyser des données CMR d'un domaine particulier : la politique. Cet article apparaît comme une synthèse des résultats obtenus et des développements mis en œuvre au sein de plusieurs projets successifs auxquels l'auteur a participé depuis 2013. Comme pour tous les articles de ce numéro, la description des aspects méthodologiques ne se présente pas comme un simple " passage obligé » en vue de l'analyse des données mais constitue une réelle réflexion scientifique visant à mettre en lumière le traitement de la complexité des données issues de la CMR. Ainsi, après avoir brièvement présenté les caractéristiques et les particularités du discours politique dans la CMR, l'auteur attache une importance toute particulière à illustrer comment ces données "sociales » ont été structurées pour constituer un corpus linguistique qu'il est possible d'explorer et d'analyser. De l'acquisition des données jusqu'à la pérennisation du corpus, les méthodes et les outils utilisés sont décrits et illustrent bien toute la complexité à prendre en compte dans l'élaboration d'un tel projet de constitution de corpus. Au cours de ce parcours méthodologique, Julien Longhi s'attarde notamment sur la définition et l'élaboration d'interfaces d'analyse du corpus qui permettent notamment l'extraction des données nécessaires aux chercheurs du domaine de l'analyse du discours. Pour illustrer cette problématique, l'auteur propose un exemple d'exploration et d'analyse du corpus centré autour de la forme 'Islam' et de ses dérivés dans le but d'explorer les différentes fonctionnalités de ce terme dans les tweets politiques et d'illustrer l'approche sémantico-discursive fondée sur un corpus complexe.

Avec l'article suivant, co-écrit par Jean-Philippe Magué, Nathalie Rossi-Gensane et Pierre Halté, nous restons dans l'univers de la communication par tweets, même si ceux-ci diffèrent de ceux présentés par Julien Longhi dans l'article précédent. En effet, le corpus présenté et analysé par les auteurs a été constitué à partir de données issues de plus de trois millions et demi de tweets «tout-venants» rédigés en français. Les données ont été collectées en décembre 2016 et correspondent à environ $10 \%$ de l'ensemble des tweets publiés sur cette période et identifiés par Twitter comme étant rédigés en langue française. Il est également important de noter qu'une méthodologie a été mise en place afin d'exclure du jeu de données analysées les tweets produits et envoyés automatiquement par des robots. À partir de ce corpus linguistique de CMR, les auteurs se focalisent sur les procédés de segmentation mis à l'œuvre dans ce genre communicationnel particulier notamment marqué par la brièveté de la communication. Dans un premier temps, les auteurs s'attardent sur l'emploi «classique » des signes de ponctuation et des connecteurs, c'est-à-dire l'emploi qui est normalement attendu dans tout texte écrit, à savoir un procédé de segmentation du texte. La suite de l'analyse se focalise ensuite sur l'emploi particulier des émoticônes et des émojis dans un corpus de tweets. Cette analyse a notamment l'objectif d'explorer les mécanismes mis en place par le scripteur du message et qui font de ces deux phénomènes des procédés 
spécifiques à la CMR. Ces deux phénomènes contribuent grandement à la complexité des corpus de ce domaine et sont un bel exemple de l'adaptation nécessaire à ces données de la part des formalismes de structuration des corpus telle que Michael Beißwenger et Harald Lüngen l'ont décrite dans leur papier.

Le dernier article conclut ce numéro en mettant en lumière un domaine auquel Thierry Chanier aura beaucoup contribué, à savoir l'apprentissage des langues médiatisé par les technologies (AMLT). Son auteure, Eva Lacroix, traite de la complexité des corpus à travers le prisme de l'apprentissage des langues. L'article débute par une discussion réflexive autour de la terminologie du domaine dans le but de souligner les similarités et les différences qui existent autour de trois termes spécifiques liés à la notion de corpus : le corpus pédagogique, le corpus réflexif et le corpus web. Dans ce papier, l'objectif principal d'Eva Lacroix est de déterminer dans quelle mesure les corpus web peuvent être amenés à soutenir et même appuyer l'enseignement et l'apprentissage d'une langue étrangère en contexte scolaire. L'auteure propose alors une discussion contrastive visant à mettre en balance les apports des corpus web comparativement à ceux des corpus réflexifs. La dernière partie, applicative, vise à détailler un scénario pédagogique ainsi que le protocole de recherche qui lui est associé. Destiné à être appliqué auprès d'un public d'apprenants collégiens de la langue allemande, Eva Lacroix illustre son discours par le biais d'exemples d'activités pédagogiques s'appuyant sur les trois types de corpus précédemment décrits par l'auteure et qui, comme elle le montre bien à travers cette étude, peuvent être employés pour explorer certaines caractéristiques d'une langue étrangère en usage.

L'ensemble de ces contributions mettent ainsi en lumière différents aspects de la complexité des corpus de la CMR et parcourent un ensemble de propositions permettant de diminuer sinon de résoudre cette complexité. Nous tenons à remercier le comité scientifique pour leur travail de relecture et espérons que ce numéro aura permis d'apporter certaines pistes de travail et de réflexion à ce domaine d'investigation qui nous semble très prometteur.

\section{BIBLIOGRAPHY}

CoMeRe Repository (2014). « CoMeRe corpora ». [website] [https://hdl.handle.net/11403/ comere].

Herring S. C. (1996). (éd.) Computer-Mediated Communication: Linguistic, Social and Cross-Cultural Perspectives. Amsterdam : John Benjamins.

Herring S. C. (2001). « Computer-mediated discourse », in D. Schiffrin, D. Tannen \& H. Hamilton (éd.) The Handbook of Discourse Analysis. Oxford : Blackwell Publishers, 612-634.

Kogkitsidou E. (2018). Communiquer par SMS : Analyse automatique du langage et extraction de l'information véhiculée (Thèse de Doctorat). Université Grenoble Alpes. [https://tel.archivesouvertes.fr/tel-01968698/document]. 
Lebart L., Pincemin B. \& Poudat C. (2019). Analyse des données textuelles. Québec: Presses de l'Université du Québec.

Mangenot F. (2008). « Pratiques pédagogiques instrumentées et propriétés des outils : le cas des forums ", STICEF (Sciences et Technologies de l'Information et de la Communication pour l'Éducation et la Formation), ATIEF, 15. [http://sticef.univ-lemans.fr/num/vol2008/05-mangenot/ sticef_2008_mangenot_05.htm].

Marcoccia M. (2004). «L'analyse conversationnelle des forums de discussion : questionnements méthodologiques », Les Carnets du Cediscor 8. [http://journals.openedition.org/cediscor/220].

ORTOLANG (2019). « Open Resources and TOols for LANGuage ». [website]. ATIFL / CNRS Université de Lorraine : Nancy. [http://ortolang.fr]

Poudat C. \& Langragin F. (2017). Explorer un corpus textuel. Méthodes - Pratiques - Outils. Louvain-laNeuve : De Boeck.

Panckhurst R., Détrie C., Lopez C., Moïse C., Roche M., Verine B. (2016). 88milSMS. A corpus of authentic text messages in French (nouvelle version du corpus ISLRN : 024-713-187-947-8). In Chanier T. (éd.) Banque de corpus CoMeRe. Ortolang : Nancy. [cmr-88milsms-tei-v1; https:// hdl.handle.net/11403/comere/cmr-88milsms/cmr-88milsms-tei-v1].

\section{AUTHORS}

\section{CÉLINE POUDAT}

Université Côte d'Azur, CNRS, BCL, France

\section{CIARA R. WIGHAM}

Laboratoire de Recherche sur le Langage, Université Clermont Auvergne, France

\section{LOÏC LIÉGEOIS}

Université de Paris, CLILLAC-ARP et LLF, CNRS, France 